\title{
Multifaceted Assessment of Ideation: Using Networks to Link Ideation and Design Activity
}

\section{Cash, Philip; Štorga, Mario}

Published in:

Journal of Engineering Design

Link to article, DOI:

$10.1080 / 09544828.2015 .1070813$

Publication date:

2015

Document Version

Peer reviewed version

Link back to DTU Orbit

Citation (APA):

Cash, P., \& S Storga, M. (2015). Multifaceted Assessment of Ideation: Using Networks to Link Ideation and Design Activity. Journal of Engineering Design, 26(10-12), 391-415. https://doi.org/10.1080/09544828.2015.1070813

\section{General rights}

Copyright and moral rights for the publications made accessible in the public portal are retained by the authors and/or other copyright owners and it is a condition of accessing publications that users recognise and abide by the legal requirements associated with these rights.

- Users may download and print one copy of any publication from the public portal for the purpose of private study or research.

- You may not further distribute the material or use it for any profit-making activity or commercial gain

- You may freely distribute the URL identifying the publication in the public portal 


\title{
Multifaceted Assessment of Ideation: Using Networks to Link Ideation and Design Activity
}

Philip Cash $^{\text {a* }}$ and Mario Štorga ${ }^{b}$

${ }^{a}$ Department of Management Engineering, DTU Technical University of Denmark, Produktionstorvet, Building 424, room 122, $2800 \mathrm{Kgs}$. Lyngby, Denmark; ${ }^{b}$ Faculty of Mechanical Engineering and Naval Architecture, University of Zagreb, Zagreb, Croatia

\begin{abstract}
Ideation is core to the innovation process, and has been the subject of study across a range of fields, from psychology to engineering. However, despite substantial progress in outcome-based descriptions of idea generation, research has often resulted in more questions than answers. For example, open questions remain with respect to the differences in behaviour related to ideation between novices and experts, the change in rates of ideation over time in different design teams, and the changing role of ideation from conceptual to detailed design. In each of these cases robust explanation has proved elusive due to difficulties in characterising the ideation process itself. This paper discusses a major new approach for elucidating ideation and its related design processes through direct observation. A novel network visualization approach is demonstrated in practice for the first time. This uses network analysis to link ideas dynamically to both the engineering context and the wider design process. This linking analysis gives substantial new insight into what drives ideation and how previously inscrutable results can potentially be explained by linking ideation into other design processes.
\end{abstract}

Keywords: creativity and innovation; human creativity; psychology of creativity; descriptive models of the design process; empirical study

To cite this article: Philip Cash \& Mario Štorga (2015): Multifaceted assessment of ideation: using networks to link ideation and design activity, Journal of Engineering Design, DOI: 10.1080/09544828.2015.1070813

To link to this article: http://dx.doi.org/10.1080/09544828.2015.1070813 


\section{Introduction}

Idea generation is the foundation of innovation and forms the basis for much of new product development and business growth (Chulvi et al. 2012; López-Mesa et al. 2011). This key role has driven an on-going focus on understanding and supporting ideation in practice (LopezMesa et al. 2009; Kudrowitz and Wallace 2013). There have been numerous studies of ideation processes in both cognitive science, and design and innovation (Dorst and Cross, 2001). However, models in cognition have typically been derived from controlled laboratory experiments using simple, isolated tasks (Kirk, 2009). These bear little similarity to ideation in the real world context where there are multiple interacting processes (Spitas 2011). Conversely, more realistic tasks have difficulty discriminating the numerous processes at work due to limitations in measurement approaches (Vargas-Hernandez, Shah, and Smith, 2010). These more 'real world' studies can struggle to generalise their results across contexts due to difficulties in drawing relations between interacting process elements (Kitchenham, 1996). This is highlighted by works such as Schlecht and Yang (2014) where ideation is a key element, but the mechanisms by which it occurs are difficult to assess due to a reliance on outcome-based metrics, such as, number of ideas or quality. In both cases, the reliance on outcome measures means that suggestions for process improvements are difficult to articulate, and more importantly, hard to assess in the real world context.

In order to address these issues, there is a need for a more holistic means for understanding idea generation and its role in design and innovation. McAdam (2004), highlights the need to address the underlying sources of knowledge creation and their link to ideation. In this context, the ideal case would be a means for analysing and linking previously isolated cognitive and design models into a more holistic picture of ideation in practice. The key to this holistic analysis is improved approaches and metrics for analysing and linking 
ideation processes. As such, this work explores a new means for understanding ideation using network analysis. The paper is structured as follows: current ideation assessment approaches are explored in both design and psychology. Next, an in-depth observation study is used as the basis for the ideation analysis. Finally, results are presented that point to new explanations for current ideation research, as well as important implications for future work.

\section{Background}

In order to better understand ideation and its relationship with design and innovation, this section builds on a critical realist perspective. Critical realism describes a system of input/outputs linked via mechanisms acting in a context (Robson, 2002). With respect to this work, Section 2.1 focuses on ideation mechanisms, which are fundamentally cognitive in nature. Section 2.2 examines the overall system and the context of these mechanisms in design. Finally, Section 2.3 examines different means for assessing the inputs/outputs. These are brought together in Section 2.4, which links ideation to the other processes in design.

\subsection{Ideation: A Cognitive Perspective}

Cognitive Psychology explores the mechanisms underpinning various human reasoning tasks (Vargas-Hernandez et al., 2010). Models of many cognitive phenomena, such as, memory, perception, and problem solving, have been developed based on controlled experiments, often using narrow tasks in order to isolate specific aspects of a process (Kirk, 2009). In this context, it is fair to say that no current model fully explains ideation and its multifaceted role

in the wider context of design and innovation. For example, The Darwinian Model (Simonton, 2003) focuses only on idea production (i.e. quantity and variety of ideas). Alternatively, the Wallas model (1926) focuses on the 'four' stages of creation: preparation, 
incubation, illumination, and verification, while the Geneplore model (Finke, Ward, and Smith, 1992) divides creative mental processes into either generative or exploratory. Further, other studies suggest that divergent and evaluative thinking are the basis for the production of creative ideas (Baer, 2003). Gabora (2002) finds this consistent with the widely held view that there are two distinct forms of thought: an associative mode perceiving metaphoric connections between correlating items in memory, and an analytic mode conducive to understanding cause and effect relationships. The first mode allows us to associate loosely related concepts and create novel thoughts, while the second gives the necessary focus to evaluate and make use of them (Gabora, 2002).

These models give useful insight into various aspects of ideation's underlying mechanisms, each forming one of a number of disparate perspectives. However, they give little guidance on their relative contribution to an overall description of the ideation system in practice (Coughlan and Johnson, 2006). In particular there is little exploration of the interaction between ideation mechanisms, context, and other work processes. For example, social context is important to individual creativity, while collaborative creativity introduces a number of additional processes e.g. interaction. As such, a holistic understanding of ideation must be inclusive of both individual and collaborative working, as well as other fundamental processes such as problem solving (Sawyer, 2003). For example, successful creative groups exhibit emergence i.e. through interaction their creative output becomes greater than the sum of their individual abilities. Although there is significant insight into the mechanisms underpinning ideation, there is a lack of work describing ideation in relation to other processes in the context of design e.g. information flow or design work. The nature of cognitive models means there is a focus on a limited number of inputs/outputs. As such, 
there is a need to better link these to the wider system perspective in design. The next section examines ideation in the context of design and innovation.

\subsection{Ideation: A Design Perspective}

When compared to cognitive research, design and innovation studies better simulate real world design by adopting a whole system perspective including varied contextual and input/output measures (i.e. less controlled environments, and more complex tasks). Here, a range of empirical methods have been used for studying the design process and its associated cognitive activities including case studies (Kleinsmann and Valkenburg, 2008), protocol studies (Christiaans and Venselaar, 2005), and controlled experiments (Reinig, Briggs, and Nunamaker, 2007; Stones and Cassidy, 2010).

With respect to ideation, investigation has focused on communication. For example, studies of teamwork (Cross and Cross, 1995), review meetings (D'Astous, Robillard, Detienne, and Visser, 2001), and data representation (Kan and Gero, 2008) have all featured prominently in the literature. While there is no consensus among researchers on a unique theory or model of design ideation at the mechanism level, each of the various theories and models available provide valuable insight at the system level. However, as in psychology, experimentation and analysis at this level is limited by the complexity of multi-faceted models involving interacting processes. For each additional variable or interaction considered, the work required increases considerably in terms of both time and complexity. For example, consider Linkography approaches, which link design 'moves' (Goldschmidt, 1990; Kan and Gero, 2008). As more processes and factors are considered the number and complexity of the Linkograph increases significantly. One result of this is that when assessing complex tasks it is difficult for results to be generalised since there is little understanding of 
the underlying process interactions (Cash, Hicks, and Culley, 2013). Further, the role and focus of ideation and creative activities change over the course of the design process (Snider, 2014).

This can be illustrated using the example of novice verses expert designers (Atman et al., 1999; Kavakli and Gero, 2002). Atman et al. suggest that experts produce fewer ideas because they have more efficient design processes, while Kavakli and Gero attribute differences to experts' more structured cognitive processes. However, these differences are difficult to trace back to their interaction with other aspects of design work as highlighted by Snider (2014).

One means for bringing these factors together is the work of Dorst and Cross (2001) on ideation as co-evolution. They characterise creative design as iteratively developing and refining both the formulation of a problem, and ideas for a solution. This is supported by reflection, synthesis, and evaluation processes, which link the 'problem space' and the 'solution space'. This model again takes a whole system perspective with cognitive mechanisms embedded in the wider context of design. Further, the model points to a key feature of studying ideation at this level - it is one of multiple processes occurring simultaneously in design work. Thus it is not sufficient to only consider cognitive ideation models in this context. In addition to the model used here (Dorst and Cross, 2001) there are a number of other works that offer holistic descriptions of design work. Notable examples include the Double Diamond of the Design-Council (2006) and the Stage-Gate process by Ulrich and Eppinger (2003).

The Double Diamond (Design-Council, 2006) gives an overview of the general progression of design work. This type of model offers an idealised description of the highlevel process, only weakly linked to the actual activity of the designer. This is not suitable 
here because it amalgamates all the processes associated with design work without having sufficient granularity to describe them individually or trace their interaction.

Ulrich and Eppinger (2003) describe the design process as a sequence of characteristic work stages e.g. detailed design. This is a common way of describing design, in the tradition of Pahl and Beitz (1996) or Clarkson's (2003) Inclusive Design model. Although this type of model is a powerful tool for shaping overall process flow and is more specific than the Double Diamond, it again fails to reflect the actual process dynamics of design activity. For example, within the detailed design stage there is a progression of different tasks and inter-related processes not captured in the overall model as found by Cash et al. (2015). In contrast, Dorst and Cross' (2001) explanation explicitly links creativity, problem solving, and design progression at the activity level. In order to understand ideation in design the interaction between these processes must be considered. However, to make this possible a final element is needed - the ability to describe the inputs/outputs and process characteristics of ideation in the wider system of design work.

\subsection{Measures of Ideation}

In order to close this systematic description of ideation in design current means for describing the system need to be considered. Without appropriate measures or analytical techniques it is impossible to decompose the processes at work in a given system. Current ideation evaluation can be broadly grouped into two categories (Nelson, Wilson, Rosen, and Yen, 2009): process-based, and outcome-based. Process-based approaches seek to evaluate idea generation based on the cognitive processes underpinning creative thought. However, due to the inherent complexity associated with these multifaceted processes (as discussed above), outcome-based approaches have become more prevalent (Nelson et al., 2009; Shah, 
Smith, and Vargas-Hernandez, 2003). Outcome-based approaches seek to evaluate the ideation process based on the concepts, or other outcomes, produced during ideation exercises. The premise of outcome-based approaches is that an idea generation technique is considered effective if its use results in 'good' ideas, where specific metrics are used to relate 'goodness' to the performance of the idea generation technique (Shah et al., 2003). In this context, several metrics have been used to evaluate the performance of idea generation techniques including, the number of ideas generated, the number of categories of ideas, the uniqueness or novelty of ideas, and the ideas' practicality. These have been distilled into a commonly recognised set of four metrics for evaluating idea generation: novelty, variety, quality, and quantity (Nelson et al., 2009; Shah et al., 2003).

- Novelty: the degree to which a given idea was unusual relative to other ideas, including those from other individuals.

- Variety: the degree to which the ideas from a single designer were dissimilar from other ideas from that designer.

- Quality: the degree to which an idea was considered feasible and met design specifications.

- Quantity: the number of different ideas generated by a designer.

It is widely believed that generating more ideas (quantity) or exploring many types of alternatives (variety) leads to better ideas (novelty, quality) (Reinig and Briggs, 2008). In previous research quantity and variety are typically considered to be process metrics, while novelty and quality are the primary outcome metrics. However, outcome metrics provide little insight into the process mechanics and thus mean it is often difficult to adequately explain why a team might produce e.g. more ideas. Further, the output measures 
highlighted above are only relevant to ideation and do not support the investigation of the overall system's progression (with interacting processes) as discussed in Section 2.2. Thus, there is a need for approaches to more easily investigate the process and process interaction perspectives.

\subsection{Research Framework}

Bringing the review together, there is a need for a more holistic interpretation of ideation if it is to be integrated into a wider understanding of design work. This builds on the fundamental mechanisms of cognitive psychology but also links to the wider processes of design work present in the real world context. Current output only measures are not sufficient to describe such a system. Thus, there is a need for an approach that can examine ideation in relation to other fundamental design processes. This transition from a monoprocess perspective to a more holistic real world perspective, based on Dorst and Cross' (2001) model, is illustrated in Figure 1. This holistic interpretation links ideation, problem solving, and design progression as three parallel, interdependant processes, with combined and separate outputs. For example, the final design artefact is a combined output, while specific ideas and activities are individual outputs. This forms the research framework for this work and allows for a holistic characterisation of ideation in design, significantly extending current mono-process models of design work. Current models provide deep understanding of numerous individual processes with little holistic understanding. Thus in order to support a more holistic interpretation this work will use a novel networking approach to link these, currently separate, process perspectives. The specific methods used for this assessment as well as the sub processes considered are outlined in the next section. In Figure 1 the inputs are considered as the initial conditions and are common across 
processes, as is the overall design context.

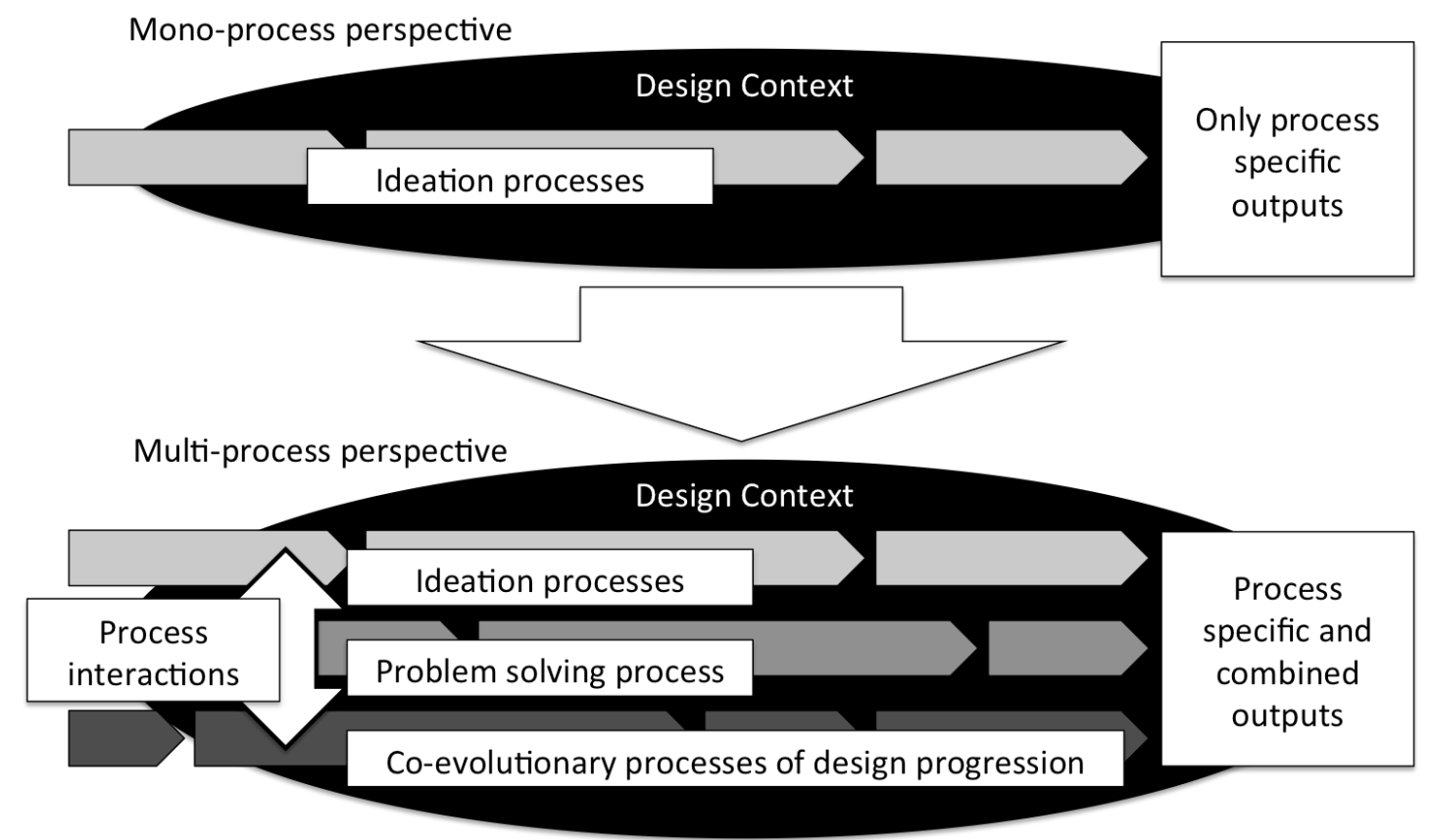

Figure 1: Moving from a mono-process perspective of ideation to a multi-process framework of ideation in relation to the wider progression of design work

\section{Method}

This section will deal with the three major methodological aspects of this work: the study design (Section 3.1), the data coding (Section 3.2), and the network analysis (Section 3.3).

\subsection{Study Design}

This work necessarily takes a theory building perspective due to the lack of multi-process theory available. As such the aim of the method outlined here is to develop a deep understanding of a small number of cases in order to both fully demonstrate the approach, and start the process of better describing ideation in the design and innovation context. This builds on the logic of single case research designs (Barzelay, 1993; Flyvbjerg, 2006). Here, generalisability is not the focus; instead understanding the interrelation between the various process perspectives is key. In order to provide a foundation for this analysis a complex 
design task was used, based on the work of Cash et al. (2013). The use of complex artificial tasks in this context is well established and has been demonstrated to be appropriate for generative studies of this type (Smith and Tjandra, 1998; Stempfle and Badke-Schaub, 2002). Further, the mixed methods approach adopted here is also well established (Hanson, Creswell, Plano-Clark, Petska, and Creswell, 2005; Kitchenham, 1996). Finally, inspiration was drawn from the work of Schlecht and Yang (2014) who also use a deep experimental observation approach in this context.

Two teams, of three participants each, were used for this study. Team 1 was selected from a previously identified population of 12 student engineers. These were in the final year of a master's level engineering program at a British university. Team 2 was selected from a population of engineers in a Small to Medium size Enterprise operating in the UK. Here there was a range of experience levels, however, all engineers shared common domain knowledge. A formal comparison of the two teams was carried out through a series of background assessments outlined in Section 4. These included an assessment of experience as well as two measures of creativity: the Kirton Adaption-innovation Inventory (KAl) test (Kirton, 1976) - measuring creative style, and the Torrance test $(1968,1998,2007)$ measuring creative thinking. Throughout, teams are labelled 1 or 2 to avoid any inference that they represent 'all students' or 'all professionals'. This type of generalisation is not the intent here.

As the focus of this work is on describing and linking the various processes associated with ideation, the study consisted of three phases: a briefing phase, a preparatory information seeking phase, and an ideation phase. In the briefing phase, participants were individually asked to complete background questionnaires before they were given information on the task. Next, in order to allow the participants to develop an understanding 
of the task, an individual information seeking period preceded the ideation activity. This aligns with studies of industrial ideation, where brainstorming sessions are normally preceded by information search activities (McDonnell and Lloyd, 2009). During the information seeking phase participants were provided with a computer and access to technical catalogues (although these were not used). In Phases 1 and 2 participants worked in isolation while in Phase 3 they were brought together to complete the brainstorming activity. At the start of each phase, the participants were given scripted instructions and a fixed time to complete each task. This information is summarised in Figure 2.

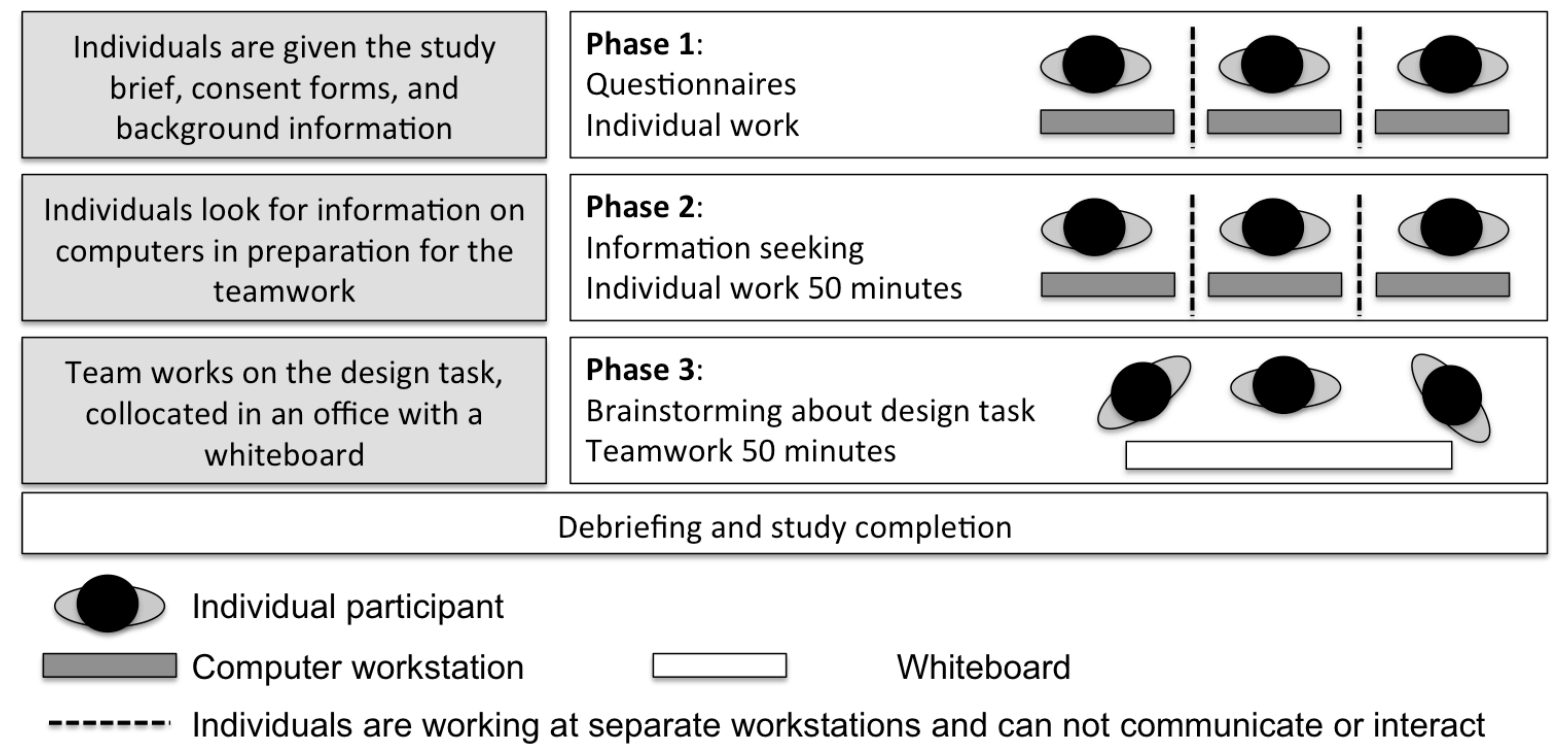

Figure 2: The study setup and process

During the whole task the brief was: "You are to design a universal camera mount for use on an aerial vehicle. The aerial vehicle (a balloon/blimp) is to be used by an amateur photographer, primarily to take still photos." In Phase 2 the participants were asked to search for additional information necessary for brainstorming about the task. In Phase 3 they were asked to brainstorm possible product ideas, focusing on mounting and moving the camera with respect to the blimp. All interactions with the participants were scripted to mitigate possible bias. Throughout the study, participants were videoed, computer activity 
was recorded, and written notes were captured via LiveScribe pens (LiveScribe, 2011).

\subsection{Coding}

In order to analyse ideation embedded in the design context a number of key characteristics were described simultaneously as outlined in the research framework (Section 2.4). Here, design is used as a focus rather than the wider scope of all possible innovation ideation contexts. In order to realise such an analysis, a protocol-based approach was adopted. Not only is this ideally suited for capturing multiple parallel information steams (Cross, Christiaans, and Dorst, 1996), it is also well established (Purcell and Gero, 1998). The protocol was based on the participants' natural conversation with no verbalisation requirements, in order to reduce observation effects. Given these general considerations this section outlines the coding schemas used to describe each of the aspects considered during Phase 3 of the study: ideation, design development, and problem solving. In each schema, codes are mutually exclusive within the groups but can otherwise exist in parallel.

Two coders were used in two stages. First, all codes were independently assessed. This gave an initial mean Kappa value of 0.72 . All areas of disagreement were then identified and discussed until a consensus was reached. This is in line with other works in this area and the achieved Kappa value supports the robustness of the coding (Bakeman and Deckner, 2003). Coding was based directly on the video recordings using the VCode software (Hagedorn et al., 2008). Thus, codes were allocated based on the definitions below and synchronised on a common timeline, illustrated in Figure 3. Using video coding in this way allowed coders to based their judgement on not only the conversation of the participants but also their interaction with the other elements in the study e.g. their logbook or the whiteboard. Thus coding represented a holistic interpretation of the definitions outlined in 
this section. Figure 3 shows a sample of the coding where P1, P2, and P3 denote which participant was speaking, and the other abbreviated labels represent some of the design development codes described in the next subsection.

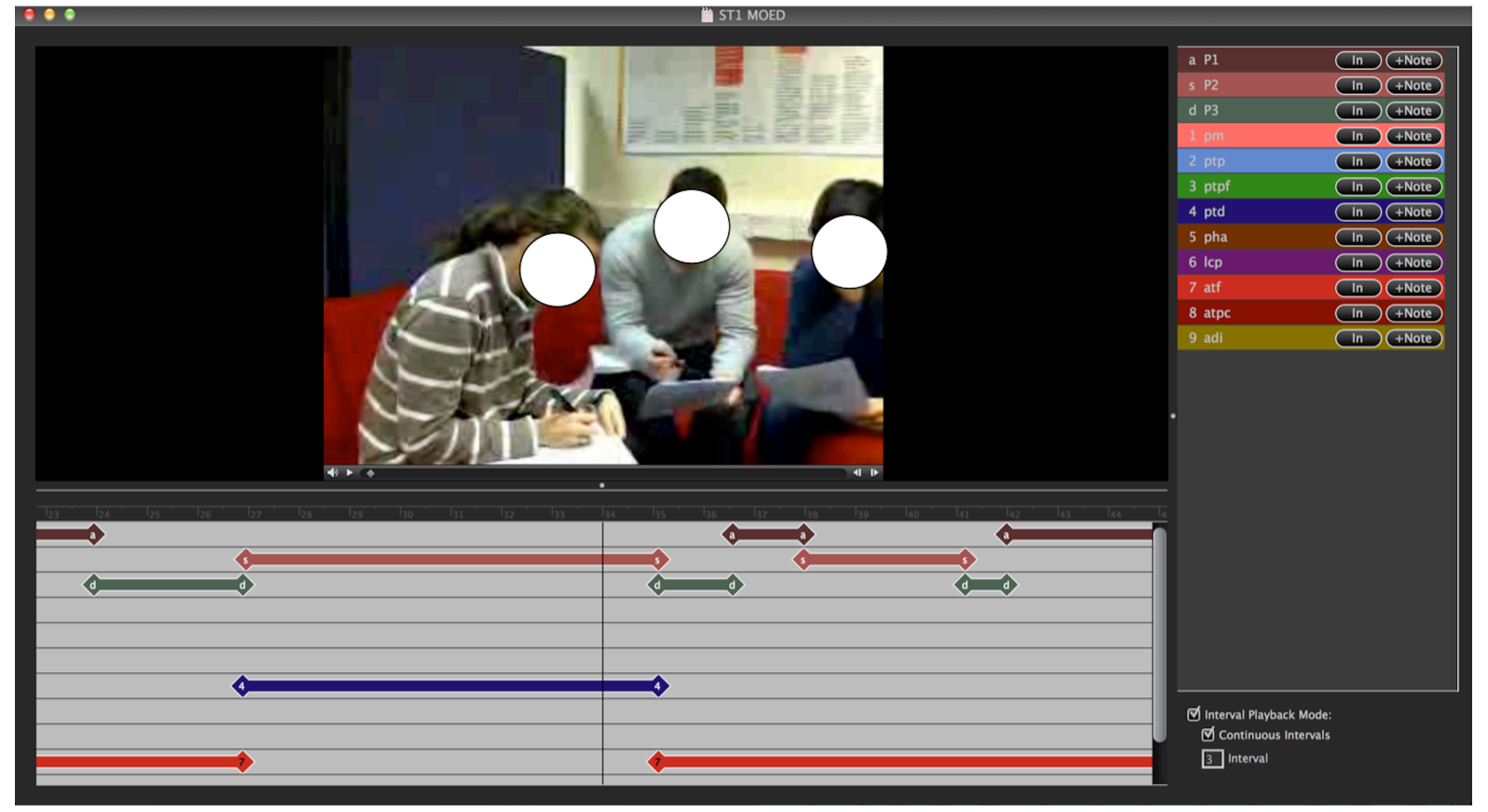

Figure 3: VCode software interface showing longitudinal codes in different categories aligned on a common timeline

\section{Ideation and Design Development}

For each idea, several attributes were coded: idea context, a team member who first articulated the idea, the information object(s) that inspired the idea, and related prior ideas. The final point was used to help evaluate idea evolution. Of these elements, the main area of contention can be found in classifying idea context. It is important to highlight that, when coding the study, ideas were considered as objects, without duration i.e. eureka moments. As such, they were recorded by a timestamp corresponding to the moment when they were first recognised by the coder. This interpretation and coding builds on the definitions used by Briggs and Reinig (2007) and Howard et al. (2010). Briggs and Reinig give a technical definition in terms of word use and purpose: "an actionable object-verb phrase that is 
presented as a potential solution to the task at hand", while Howard et al. focus on the design role: "a generative proposition of function, behaviour or structure".

Idea context relates a particular idea to the design (product) itself, design attributes, and the life cycle phase of the future product being discussed. As an inspiration for coding, the MOED ontology (Ahmed and Storga, 2009; Storga et al., 2010) was used. This defines a generic taxonomy of subjects tackled in engineering design activity. This taxonomy offered a significant level of detail when coding the design activity - beyond that of higher level frameworks, such as, C-K (Hatchuel and Weil, 2003), or Function-Behaviour-Structure (Gero and Kannengiesser, 2004). As MOED is well established and extensive, the full classification is not repeated here, however, it is important to note that the codes are distributed over three high-level domains: the physical object, the physical process, and the abstract attributes. All MOED codes are mutually exclusive. As such, the idea context was coded according to the codes listed in Table 1.

Information objects that inspired ideation were considered as two types direct or indirect. Direct inspirations are those that were used unmodified from the original information objects e.g. "it was stated like that in this source". Indirect inspirations are those that were used in a modified form compared to original, or previous experience of the team member. 
Table 1: Idea context codes based on MOED (Ahmed and Storga, 2009). Definitions are included here but further detail can be found in the work of Ahmed and Storga (2009)

\begin{tabular}{|c|c|c|}
\hline Group & Code & Definition \\
\hline \multirow{6}{*}{$\begin{array}{l}\text { Physical } \\
\text { object }\end{array}$} & Material & A tangible substance that goes into the makeup of a product \\
\hline & Assembly & $\begin{array}{l}\text { A group of components that fit together to form a self-contained structural } \\
\text { and functional unit }\end{array}$ \\
\hline & Component & $\begin{array}{l}\text { A product that is one of the individual parts with a specific task in realization } \\
\text { of the technical function, and of which an Assembly is made up }\end{array}$ \\
\hline & Form feature & An individual part of an component's form \\
\hline & $\begin{array}{l}\text { Technical } \\
\text { solution }\end{array}$ & $\begin{array}{l}\text { A product that in use and life cycle phases realizes necessary effects that } \\
\text { satisfy the user requirements }\end{array}$ \\
\hline & Product family & A collection of different variants of the same kind of product \\
\hline \multirow[t]{6}{*}{$\begin{array}{l}\text { Physical } \\
\text { process }\end{array}$} & Planning & $\begin{array}{l}\text { An intentional process of drawing up the design issues and plans for } \\
\text { development of a product }\end{array}$ \\
\hline & Designing & $\begin{array}{l}\text { An intentional process of working out product characteristics based on the } \\
\text { required functions, and solving design issues leading to a product description }\end{array}$ \\
\hline & Manufacturing & $\begin{array}{l}\text { An intentional process of making components from raw material and } \\
\text { assembling them together }\end{array}$ \\
\hline & Distributing & $\begin{array}{l}\text { An intentional process of transporting, selling and installing a product from a } \\
\text { producer to a customer }\end{array}$ \\
\hline & Exploiting & $\begin{array}{l}\text { An intentional process of putting a product into service and make it work for } \\
\text { the particular purpose of fulfilling its function }\end{array}$ \\
\hline & Disposing & $\begin{array}{l}\text { An intentional process of processing used products for use in creating new } \\
\text { products }\end{array}$ \\
\hline \multirow{9}{*}{$\begin{array}{l}\text { Abstract } \\
\text { attribute }\end{array}$} & Function & What product is manufactured and used for \\
\hline & Shape & The spatial characteristic of product defined by its surface area \\
\hline & Dimension & The magnitude of product in a particular direction \\
\hline & Tolerance & A permissible difference of nominal dimension of the product \\
\hline & $\begin{array}{l}\text { Manufacturing } \\
\text { method }\end{array}$ & $\begin{array}{l}\text { A particular method applied in fabricating and assembling a component or } \\
\text { assembly }\end{array}$ \\
\hline & Surface texture & The totality of the micro geometrical incorrectness of an component's surface \\
\hline & $\begin{array}{l}\text { Structural } \\
\text { characteristics }\end{array}$ & A manner of designing the product and the arrangement of its parts \\
\hline & $\begin{array}{l}\text { Life cycle } \\
\text { requirements }\end{array}$ & Attribute of a product required by different life cycle stages \\
\hline & $\begin{array}{l}\text { Environmental } \\
\text { requirements }\end{array}$ & $\begin{array}{l}\text { Attribute of a product required by the totality of the surrounding conditions of } \\
\text { its physical environment during its life cycle }\end{array}$ \\
\hline
\end{tabular}

\section{Design Problem Solving}

With the ideas and design context coded it was next necessary to consider the other process

of interest during the session. Here, problem solving was characterised by eight fundamental

activities. These were drawn from the work of Wasiak et al. (2010) and have been previously

validated by Cash et al. (2013). These codes are summarised in Table 2 and linked to the

specific colours used in the network by the key attached to Figure 6 . 
Table 2: Problem solving codes

\begin{tabular}{|l|l|l|}
\hline Group & Code & Definition \\
\hline \hline \multirow{4}{*}{$\begin{array}{l}\text { Problem } \\
\text { solving }\end{array}$} & Goal setting & Identifying where the design is and where it needs progressing to \\
\cline { 2 - 3 } & Constraining & Imposing boundaries with requirements and desirables \\
\cline { 2 - 3 } & Exploring & Discussing possibilities and ideas invoking suggestions \\
\cline { 2 - 3 } & Solving & Involves searching, gathering, creating, developing solutions \\
\cline { 2 - 3 } & Evaluating & Judging the quality, value and importance of something \\
\cline { 2 - 3 } & Decision making & Considering evaluation and possible compromises to form decisions \\
\cline { 2 - 3 } & Reflection & Reflecting upon a design decision or process already adopted or occurred \\
\cline { 2 - 3 } & Debating & Discussing opposing views \\
\hline
\end{tabular}

\subsection{Network Analysis}

In order to relate the elements identified above a network analysis approach was adopted.

This is currently unique in its application to ideation. In order to carry out this analysis, the protocol dataset was further refined via the following steps. Once complete this process presents the data in network form ready for further analysis and refinement.

1. The data must be defined taxonomically and able to be related to each other.

2. The nature of the relationships should be established for the network analysis.

3. Mathematical modelling is used to translate the recorded data into the network structure.

Elaborating these further, each step is described below. First, the coding schema described in Section 3.2 provided the taxonomy for the network. Each code is used as a graph node label, as illustrated in the example network sample shown in Figure 4. Note that 'idea' nodes are assigned unique identifying numbers in order to differentiate concepts. Nodes are defined in two domains: problem solving activity type, and ideas with respect to context.

Second, the links between nodes were defined. Although relationships between variables were surmised based on theory (Section 2), the relationships used for the initial network analysis were defined as: temporal relations link activities, and mapping relations link activities to ideas, and idea context, based on Cash et al. (2014). For example, if a 
participant is exploring and at the same time generates an idea then these two nodes would be mapped (dashed line in Figure 4). Similarly two activity nodes are linked if they follow in time sequence (solid line in Figure 4). For example, if a participant is exploring and then changes to goal setting. As such, temporal links are directional - progressing in time, while mappings are non-directional.

Finally, these features were transformed into a network structure based on the process described by Cash et al. (2014). This was adopted as it constitutes the current state of the art in this type of analysis with respect to design activity. Each session was split into a finite number of steps $i$ with the corresponding network elements added to each. The network tool then automatically created the links based on the rules outlined above. Here, the overall shape provides a visualisation of the node's connectedness. This can take two forms, simple patterns where nodes are only linked temporally (see Figure 6); or clustering where nodes are linked both temporally and using additional factors (see Figures 4 and 7), such as context. In the latter case nodes that are not directly linked temporally can be connected via other common features, for example, both being related to the discussion of the technical function. Nodes cluster based on the number of links between them e.g. the purple grouping at the bottom of Figure 4. Further, the tool automatically assigns colours to these clusters to aid interpretation. These colours are arbitrarily assigned - only denoting that the nodes are clustered e.g. the green/red/purple colouring in Figure 4. These colours can change during network growth as new clusters form or split (see Figure 7 as an example). Overall the key elements in the network are the clusters and node connections. The OrganicViz tool (Stankovic et al., 2012) was used to create the network visualisations used throughout this work. 


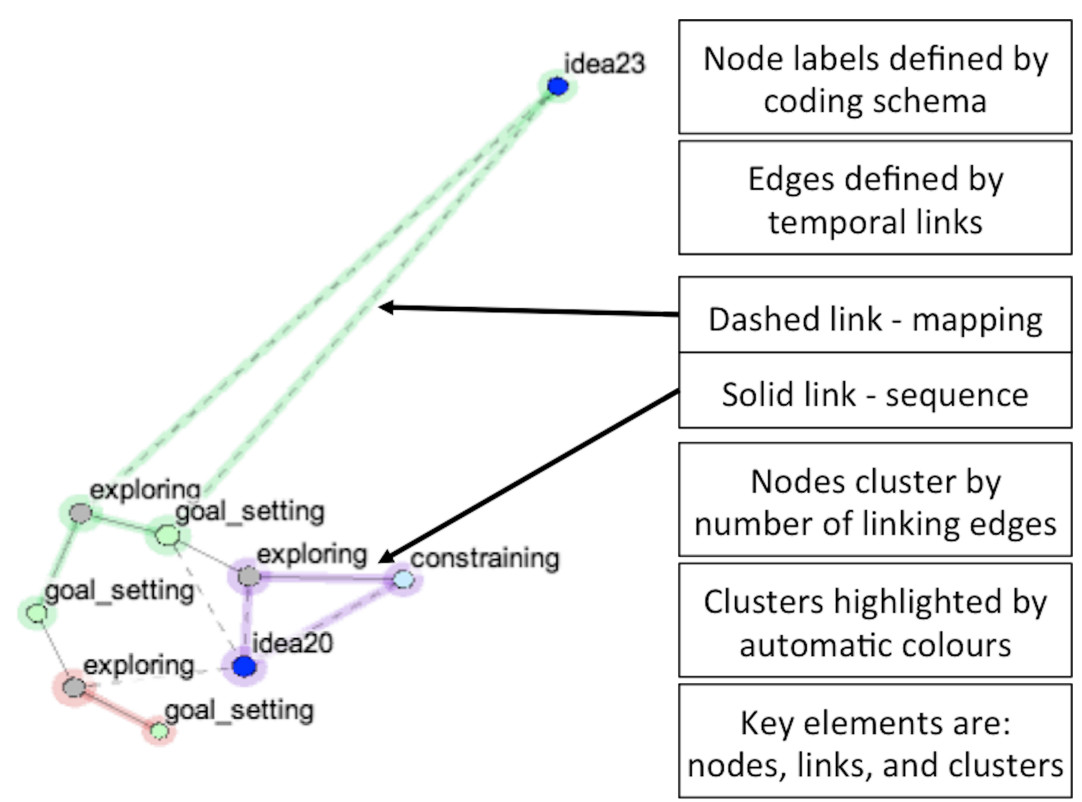

Figure 4: Example network sample highlighting key features

\section{Results and Discussion}

As the main focus of this work is the development of a more holistic assessment of ideation the complexity is gradually built up, with each section adding a new element to the whole. This is complemented throughout by quantitative and qualitative results in order to illustrate the approach, and describe the ideation observed in the study.

\subsection{Basic Ideation}

The first element to consider is the baseline comparison of the teams themselves. Four baseline measures were used: education, industrial experience, Kirton Adaption-innovation Index (KAI) for creative style (Kirton, 1976), and the Torrance test for creative thinking (Torrance, 2008). In Team 1 (students) distribution of all measures was homogeneous and close to the population norm. In Team 2 (professionals) there was a range of results with one individual displaying highly innovative (KAI 136) and creative tendencies (Torrance 122). Further, one member was educated to $\mathrm{PhD}$ level. As such, the team was more heterogeneous. These results are summarised in Table 3. 
Table 3: Baseline data

\begin{tabular}{|l|l|l|l|l|}
\hline & Education & Experience & KAI & Torrance \\
\hline \hline Student 1 & Masters Engineering & 12 months & 100 & 96 \\
\hline Student 2 & Masters Engineering & 13 months & 104 & 108 \\
\hline Student 3 & Masters Engineering & 12 months & 100 & 113 \\
\hline \hline Professional 1 & Masters Engineering & 42 months & 136 & 122 \\
\hline Professional 2 & Masters Engineering & 49 months & 106 & 103 \\
\hline Professional 3 & Masters Engineering, Phd & 24 months & 89 & 98 \\
\hline Standard 50th percentile population means & $\mathbf{9 6}$ & $\mathbf{1 0 1}$ \\
\hline
\end{tabular}

Based on the results in Table 3 Team 2 would intuitively be expected to be more creative but have greater difficulty in coordination and communication. However, current literature suggests that the greater experience of Team 2 will temper their creativity and make them generally more focused on the problem at hand (Atman, Chimka, Bursic, and Nachtmann, 1999; Kavakli and Gero, 2002). This interpretation is supported in Figure 5, which shows the ideas generated over time for the two teams. The teams develop a similar number of ideas over the first half of the session, but in the second half Team 1 increase their output and move away from Team 2. Although this conforms with current literature (Howard et al., 2010), there are a number of unanswered questions. First, why does the rate of ideation change part way through the session, and are the teams truly similar in their ideation process. Both of these have proved extremely difficult to answer using current research approaches and, as such, highlight the need for the new approach described here. 


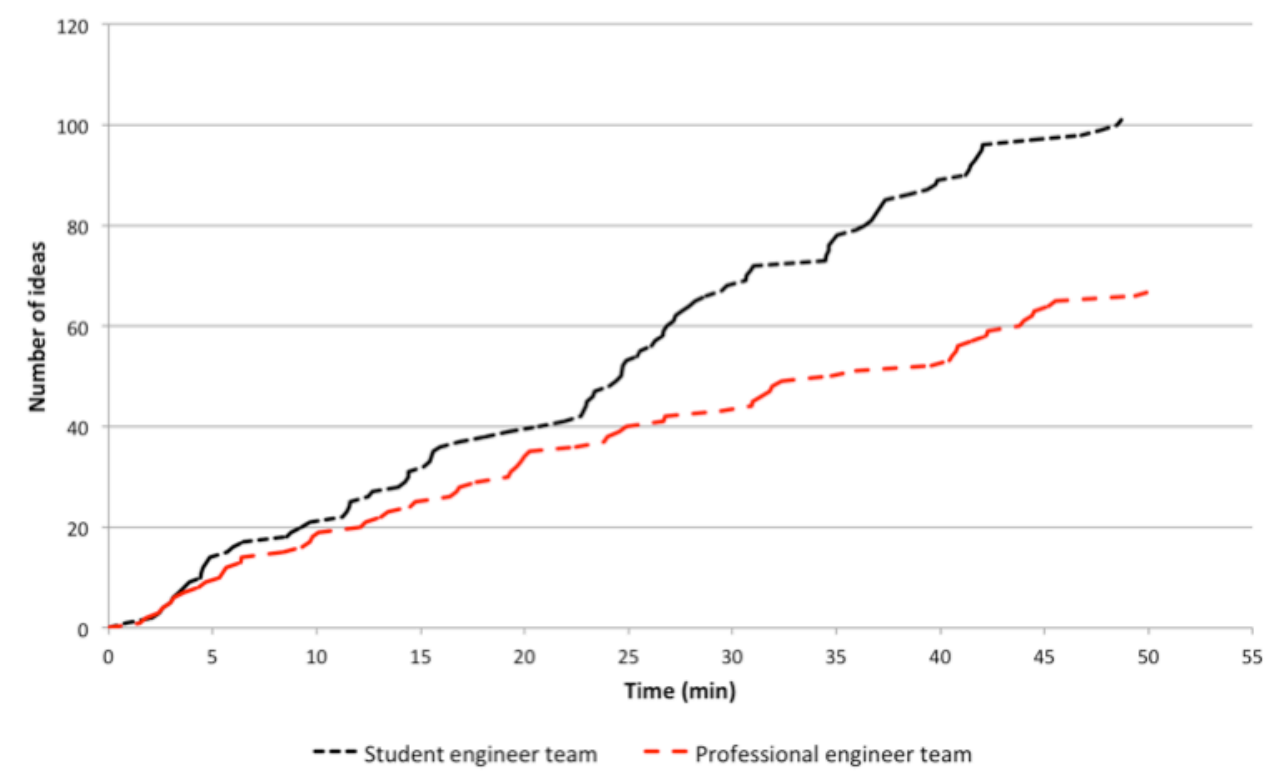

Figure 5: Ideation over time only considering idea generation

\subsection{Ideation and Problem Solving}

The next element to be considered is the relationship between problem solving and ideation. Figure 6 depicts the combined networks generated for the two teams. Here, the features of note are the patterns of ideas and the sequential progression of activities. Ideas have been highlighted in blue, while the various activities are defined in the key for Figure 6 . Both networks show a linear progression of activities because clustering was not used in this visulisation, instead activities are only grouped by temporal connection. Clustering requires the introduction of a third element, context (or another variable), to link temporaly disasociated activities, as discussed in Section 4.3. The large scale structure of the networks in Figure 6 (the overall circular sweep) is thus arbitrary, simply allowing the linear progression to be displayed efficiently. Network growth is not illustrated in Figure 6 as it is a purely sequential progression from Session start to Session end. 

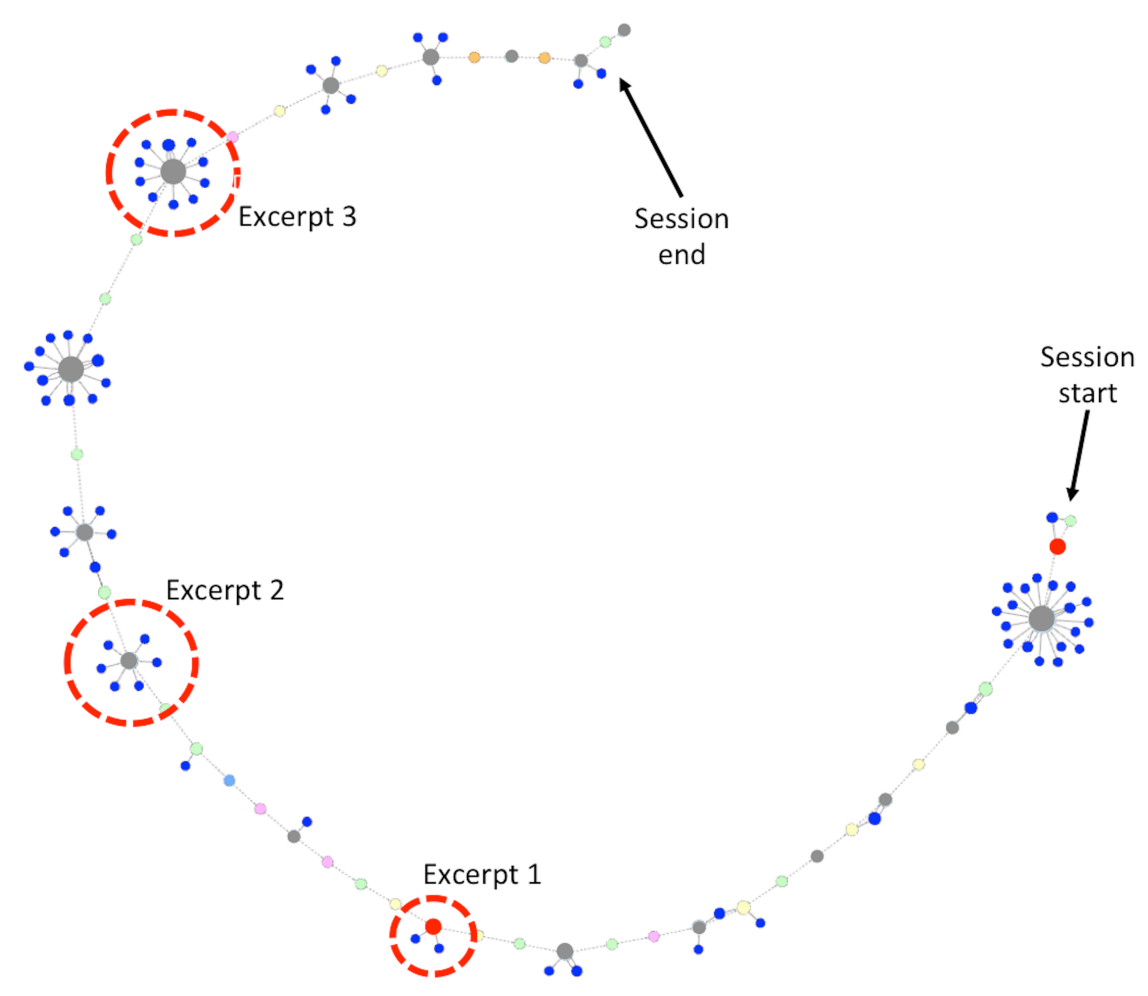

Figure 6a: Team 1

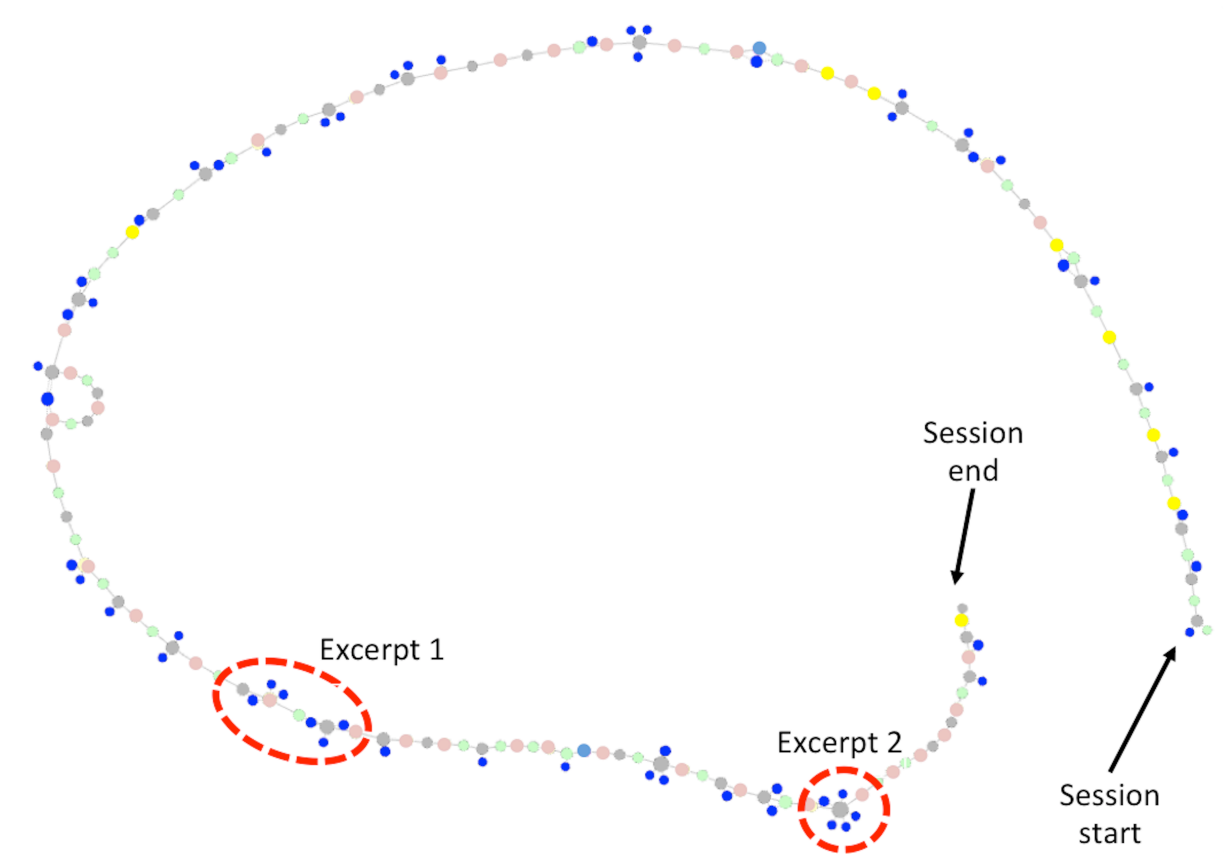

Figure 6b: Team 2

goal setting

evaluating

\begin{tabular}{l|l|l} 
solving & $\begin{array}{l}\text { exploring } \\
\text { decision making }\end{array}$ & constraining \\
ridea
\end{tabular}

Figure 6: Problem solving activity and ideation for the two participant teams

Comparing the two teams in Figure 6, the first apparent difference is in the patterns of ideas.. In Team 2 the network emerges as a highly linear progression of individual 
idea/activity couplings with only one group of ideas larger than three. In contrast, Team 1 has a substantially different character, with four midsized idea groups, and three larger than six ideas. Here, the majority of the teams' ideas are found in these patterns. This highlights a major difference in how the two teams are ideating. Figure 6 suggests that Team 2 was more rapidly iterating between problem and solution related activities, while Team 1 spent more time ideating around specific problem elements. This is supported by the average duration of the activities in each team (all in seconds): Team 1 mean $=20.2, \mathrm{SD}=41.6$; Team 2 mean $=$ 13.7, SD $=16.2$

Exploring this further, the way in which the teams ideation activity developed also differed on the small scale. There are distinct differences between the first portion of the study (where the teams were ideating at a similar rate (Figure 5)) and the second half (where the teams diverged). Throughout, this progression is described with respect to the preactivity, the activity/idea, and the post-activity. In the first portion of the study contrast Team 2's (bottom), and Team 1's (top) progression. The progressions outlined below represent $81 \%$ of the activity sequences for Team 2 and $44 \%$ for Team 1 . No other individual progressions represented more than $6 \%$ of Team 2 and $12 \%$ of Team 1's activity sequences.

Goal setting/constraining $\gg$ exploring + multiple ideas $\gg$ constraining/goal setting (Team 1 )

Evaluating/goal setting > exploring + idea > goal setting/evaluating (Team 2)

These two progressions highlight a key difference between the teams. Team 2 use evaluation as a springboard for ideation, which leads into further evaluation or goal setting. This closely aligns with co-evolution where each successive idea forms the basis for furthering the teams understanding of the problem or solution spaces. In contrast, the Team 1 adopts a more constraint-focused approach. They define specifications or requirements 
that they explore through ideation. This is carried out concerning an initial discussion of requirement areas, and thus progresses arbitrarily. This corresponds to a process, which can be summarised as - identify constraint/requirement, generate ideas around this requirement, move to next requirement.

In the second period, where the teams diverge (Figure 5), the differences in ideation progression are illustrated below (Team 1 top, Team 2 bottom). The progressions outlined below represent $67 \%$ of the activity sequences for Team 2 and $30 \%$ for Team 1 . No other individual progressions represented more than $6 \%$ of Team 2 and $10 \%$ of Team 1 's activity sequences.

Goal setting $\gg$ > exploring + multiple ideas $\gg$ goal setting (Team 1 )

Goal setting/evaluation >> exploring + idea >> constraining/goal setting (Team 2)

Here the two teams seem superficially similar, despite the difference in ideation rate. However, they adopt fundamentally different ideation approaches within these progressions. As in the first period Team 2 continues to iterate rapidly between problem and solution spaces with each progression associated with just one or two ideas. In contrast Team 1 identifies a single requirement, then ideates extensively before moving to the next goal setting requirement. This approach means that a large number of ideas are generated. However, there is little critical reflection as to their impact on the design, as indicated by the lack of evaluation activities. This is supported by the difference in the mean number of ideas produced per activity in each team: Team $1=1.6$, Team $2=0.40$. Looking closer at Team 2 there are two distinct modes. The first couples goal setting and evaluation, while the second couples constraining and goal setting. In this way Team 2 are focused on progressive evaluation, continually working to deepen their understanding of the problem/solution 
through their ideation.

Further, it is possible to pick out distinct qualitative differences in the activity of the teams by contrasting superficially similar features in the two networks. First, consider the individual idea/activity situation (Excerpt 1, Figure 6). Throughout, the excerpts have been editorialised using brackets. In the first Excerpt the teams show similar ideation approaches, starting with a question e.g. how to close the shutter. This then leads to a solution proposition idea followed by an explanation. Both teams then seek to clarify if this meets the requirements, although this is more extensive in Team 2. Finally, in Team 2 this leads to a reformulation of the original problem to further probe the problem space e.g. instead of controlling the shutter, could the camera be autonomous. Conversely, participants in Team 1 state that the idea would fulfil the requirement without further judgement; this then prompts them to select the next problem. Overall the discussion of each idea is more focused in Team 2, with feasibility being evaluated at each juncture. This also helps to explain why Team 2 tends to produce single ideas alternating with evaluating or goal setting activity.

Excerpt 1: Team 2, 1429-1493 seconds

1 Tom How are we going to pull the shutter?

2 Bill I found some things that do that [references research documents], there is a remote shutter release

3 Tom do you know mechanically how it works? I have an old fashioned one with a pump...

4 Bill you don't need a mechanical...

5 Tom ... [explains mechanism at length]

6 Bill but what you get now is radio remote controls that just trigger the camera

7 Tom I have one but they are specific to cameras and the range is poor

8 Bill This one [references research documents] fits a number of camera brands

9 Tom ...so they are working with infrared?

10 Bill wireless RF

$11 \mathrm{Jim}$ so we are assuming that the user controls when they want to take a photo, there are other options

12 Tom yeah a timer

13 Jim or just continuous snapshots 


\section{Excerpt 1: Team 1, 762-834 seconds}

1 Bob we haven't got an angle for how far it needs to go down

2 Jane I think it needs to go 90 degrees

3 Tim ... I am not sure if we want one, but if we got one really wide angle lens

4 Bob limit the actual range of rotation?

5 Tim if we get the camera pointing in this direction [sketches camera position in logbook], then the lens could take in this view [completes sketch], so we can just pivot around one point, that could be a bit lame [vernacular for problematic]

6 Jane that is true, it would do the entire requirement [all agree]

7 Bob what else do we need to think about...

[Leads into ideation around control of the blimp]

Next consider the larger patterns of ideas (circa five) generated by the two teams (Excerpt 2,

Figure 6). In this context, the difference between the two teams starts to become more pronounced. Here Team 2 again adopts the reflection and judgement driven approach, first refining the requirement before proposing and judging a small number of feasible solutions. Conversely Team 1 first identifies a problem before moving directly to the next issue and ideating about that. Here, there is only a brief mention of feasibility and no concept judgement before they conclude that the area is closed and move to the next.

\section{Excerpt 2: Team 2, 2640-2733 seconds}

1 Bill we can use other masses to help with positioning. [pause]

2 Bill in fact you could have some sliders. Maybe there are some sliding weights you could use, with a reasonably fixed mount, or maybe two or three possible mounting positions.

3 Tom What about a plate with lots of screw holes [general agreement], or positions that you attach the plate with screw holes too. That is just a bit of a messy solution.

4 Bill A plate does deal with two axis, we still have another one. But you could do that with spaces, or with a telescopic pole. We could have other sliding weights that move on the sphere to position it [long pause, while the team look at the working sketch on the whiteboard]

5 Bill We shouldn't forget about using strings for positioning either, because the rollers don't really work.

6 Jim We haven't got that written down. [references whiteboard]

7 Bill We have a lot of string, look these are strings [references the working sketch on the whiteboard]. Because you only need two positioning strings wrapped round a drum, and you just drive the drum one way or the other and that will rotate the sphere 
8 Jim Are you talking about shortening the length of the string here [references whiteboard, general agreement]

9 Bill Oh yeah, we could suspend it here [references the working sketch on the whiteboard] and then we could control these...

[Leads into a comparative discussion of the two concepts' pros and cons]

\section{Excerpt 2: Team 1, 865-954 seconds}

1 Tim can we expand a bit more on the mechanics, so when you [indicating Jane] said the ball, I had an idea of like [sketches in logbook], a ball like this where the camera is attached here [points Jane to sketch]

2 Bob like a ball and socket kind of joint [writes note on whiteboard] power?... electrical

$\begin{array}{lll}3 & \text { Jane } & \text { solar power } \\ 4 & \text { Bob } & \text { wind, it will definitely get that } \\ 5 & \text { Tim } & \text { batteries } \\ 6 & \text { Bob } & \text { batteries [makes note on whiteboard] } \\ 7 & \text { Tim if we do use batteries I think lithium ion } \\ 8 & \text { Jane } & \text { it does say [references the design brief] one and a half hours per charge } \\ 9 & \text { Bob can be charged when it is up there [in flight], I think that area [of discussion] is } \\ & \text { done now }\end{array}$

Finally, a unique feature of Team 1 was the large pattern of ideas (greater than 5) (Excerpt 3,

Figure 6). This reinforces the pattern illustrated in Excerpt 2. Team 1 starts with a problem, which results in a number of ideas, one of these then directly inspires the next round of ideation. This results in the rapid generation of ideas on a number of topics without reflection or feedback.

Excerpt 3: Team 1, 1525-1653 seconds

1 Jane I have another problem that they sometimes include is the things holding the camera could get in the shot, like the blimp

2 Tim ok, [makes note on whiteboard] any other...

3 Bob see what we can do to eradicate that, by limiting the angle of rotation

4 Jane you could have instant feedback to the user so they can see if something is in the shot and move the camera

5 Tim kind of like user controlled

6 Tim lets put Photoshop

7 Jane you could have the camera lower with the wires further away from the blimp so it is less likely to be in the shot

8 Bob that could be variable as well

9 Tim I think it is more interesting how this comes round [references sketch on whiteboard]

10 Bob waterproof it maybe 
11 Jane the angle of the sun, in the morning the sun is lower so could shine directly into the camera, while at mid day it would be above, so I don't know if that would affect the camera support, it is something to take into consideration

\subsection{Ideation, Problem Solving, and Design Development}

Adding the design development element transforms the network representations by allowing clustering and illustrates some of the key differences between the teams' ideation approaches. Here, Team 1 adopts a more linear approach, exploring each element at length before progressing to the next. This is illustrated by the sequential development of problemsolving activites associated with ideation context: functional and life-cycle requirements, technical function, and technical solution. A hierarchical clustering algorithm (Newman, 2004) was applied in order to analyse the community structure of the network. The algoritm detects groups of nodes that have a high density of edges within them, with a lower density of edges between the groups. This also helped explain how different problem-solving activities occuring in different parts of the study were related to particular ideation contexts. This progression is highlighted in Figure 7a. Here, the growth of the noted network clusters is shown in steps 1-6 above the final network. In steps 1-4 three clusters develop, In 5 and 6 one new cluster is formed, and one existing cluster splits in two. Each of these clusters is associated with a different ideation context. Note that clusters are allocated arbitrary colourings to aid interpretations as explained in Section 3.3. This progession represents the development of the participants design activity over the course of the session, shown at evenly distributed intervals. This sequential development of clusters related to different design aspects greatly reduces the number of iterations between problem and solution domains and corresponds with less reflection on the technical implementation and feasibility, as noted in the previous section. This is indicated by the size of the idea nodes (here reflecting their common context) in the final network, at the centre of each cluster. 
Node size indicates the node degree - the number of connections to that node. Thus, large nodes distributed across clusters in this way denotes a step by step approach in line with the clustered ideation observed in Figure 6.

In contrast, Team 2 focused on the technical solution as illustrated in Figure 7b. This resulted in a dominant central cluster about which all activities orbit. This translates to a process of rapid iteration, where the activity is continuously linked back to the development of the technical solution. This is shown by the development of linear strings of activity that all link back to one idea context in the central blue cluster. In this manner Team 2 complete a large number of iterative loops exploring various aspects of the problem space while retaining a focus on developing the solution. This rapid assessment and linking of different technical elements is illustrated in the example progression in Figure 7b. This is of particular note because the design elements considered by both teams are similar but are dealt with in fundamentally different ways. Again, the cluster colourings are only used to aid interpretation. 


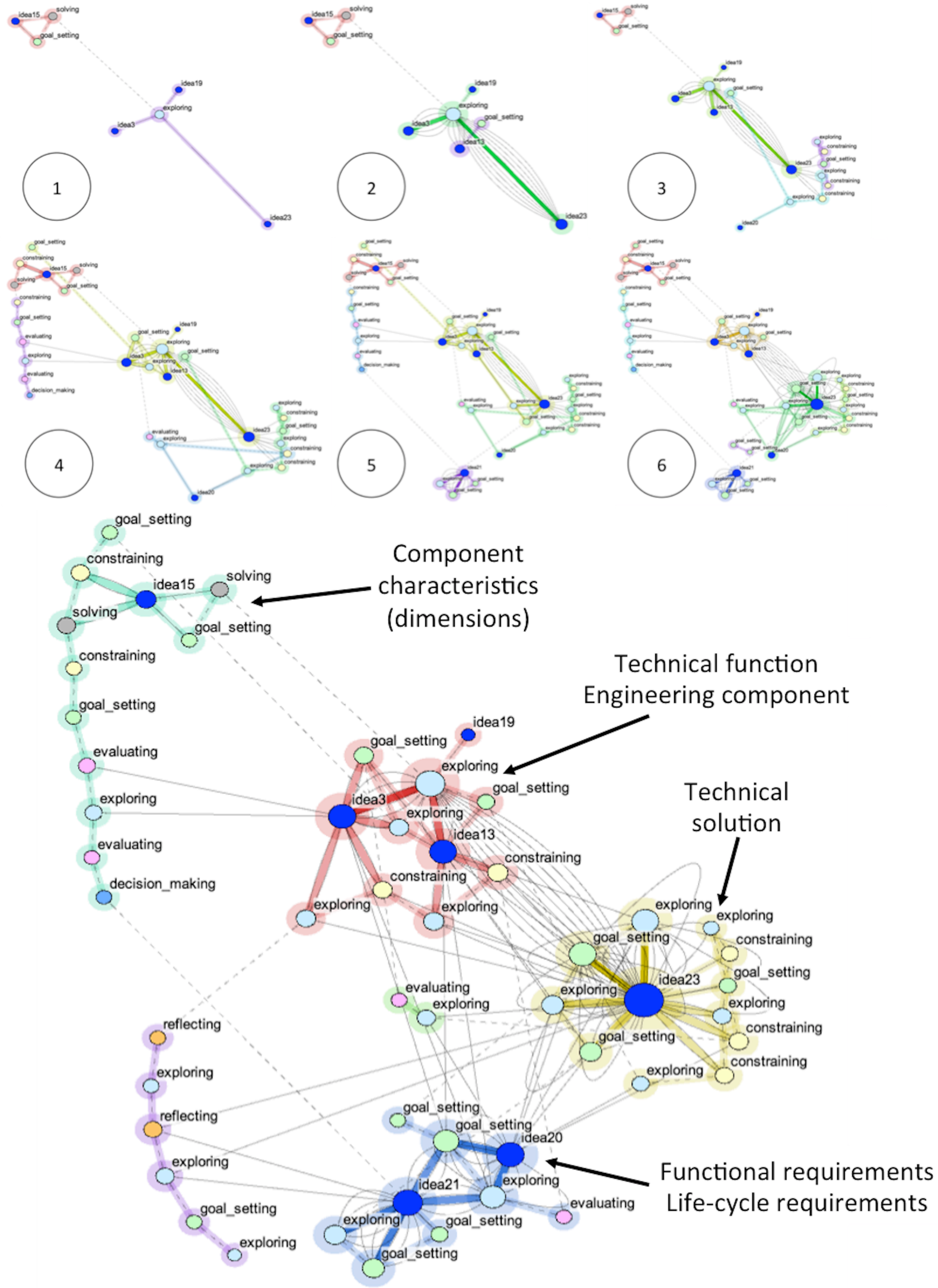

Figure 7a: Network growth and final network for Team 1 structured by ideation context 

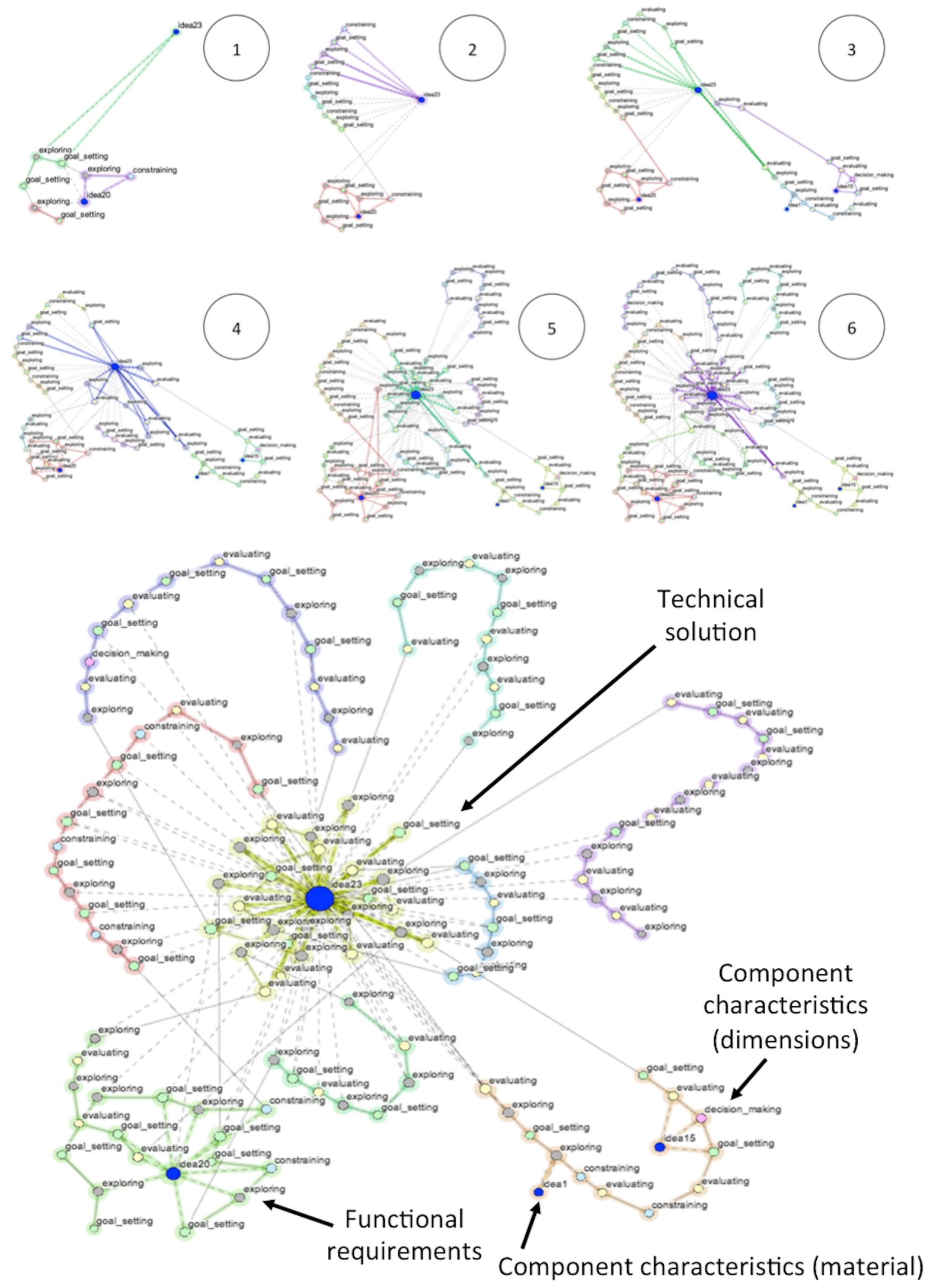

Figure 7b: Network growth and final network for Team 2 structured by ideation context

Figure 7: Problem solving activity and ideation context for the two participant teams 


\subsection{A Holistic View of Ideation}

The results discussed throughout this section have significant implications in two regards how ideation may be interpreted, and how current models of design explain ideation.

Focusing first on current models of ideation and design work, there are none - to the authors' knowledge - that explicitly integrate design processes and idea generation. However, the results described here provide a platform for refining these two concepts to give a new more holistic perspective on real world ideation. In particular, two distinct ideation patterns emerge from the analysis. The first type, employed by Team 1 , is illustrated in Figure 8a. Here, the team progressed methodically through problem solution iterations with a focus on producing many ideas at each point, without reference to the other domain. Contrast this to the second type, employed by Team 2 and illustrated in Figure $8 \mathrm{~b}$. Here, the team focused on the development of the technical solution as the main outcome of the design session. As such, they generated ideas through rapid iteration between problem and solution domains. This resulted in a continuous refinement of both domains with a smaller number of ideas generated during each iteration. This evolutionary approach to ideation has been previously highlighted as important in the design and innovation context by Tang (1998), and Lee and Lee (2004). Figure 8 also shows network segments indicative of the two progression types.

This differentiation in process interactions highlights a key element of the network analysis method in the context of ideation. The network allows multiple processes to be considered holistically, a key missing element in current approaches (Nelson et al., 2009). Further, this lets the researcher brings together individual and group elements by allowing protocol data to be interrelated with respect to various processes, including social or individual elements (Sawyer, 2003) As such, this poses an important new means for 
measuring and interpreting ideation in design and innovation, which is complementary to current outcome based measures. In particular this links to works where ideation is associated with wider processes, such as, user involvement (Goodman-Deane, Langdon, and Clarkson 2010). Further, the additional analytical dimensions allowed by this type of analysis provide new means for assessing the final ideation outcomes, thus directly enhancing current outcome-based measures, such as, Shah et al.'s (2003) four metrics.

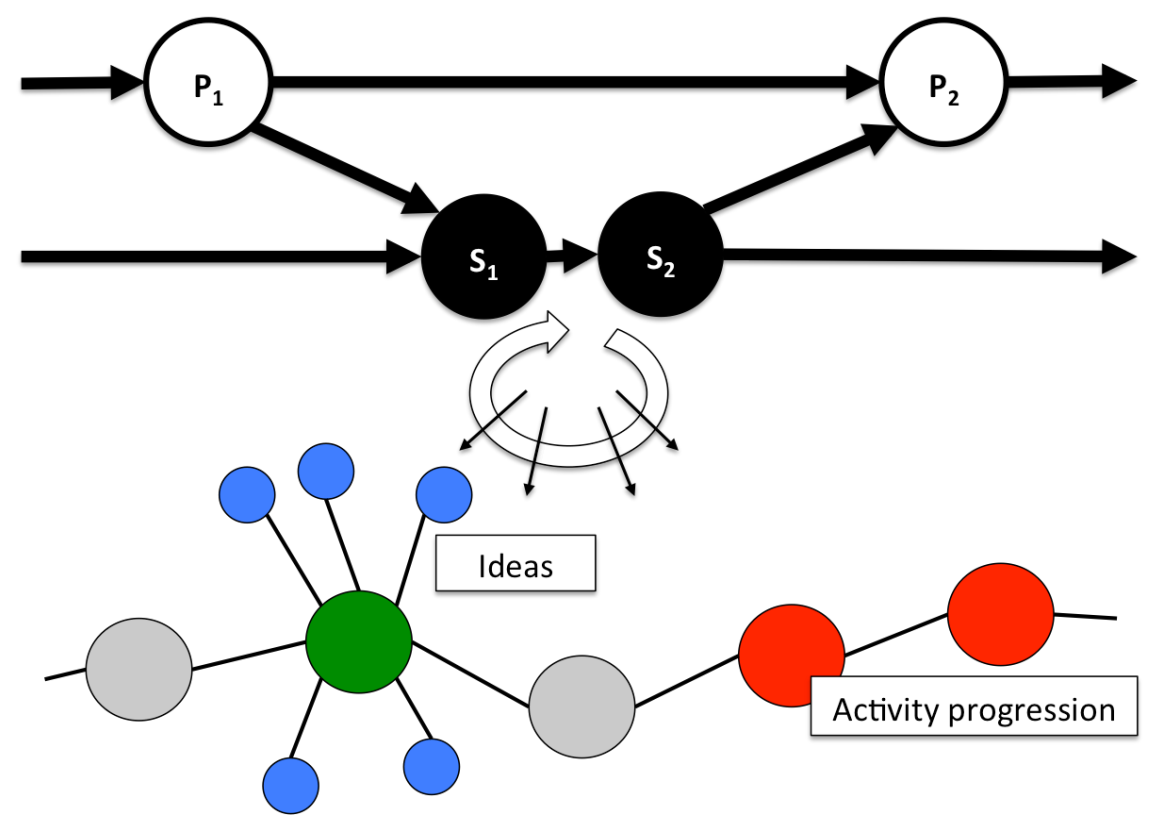

Figure 8a: Decoupled ideation produces numerous ideas without feeding into the coevolution process. This also shows a much slower progression of the co-evolution process with less iteration.

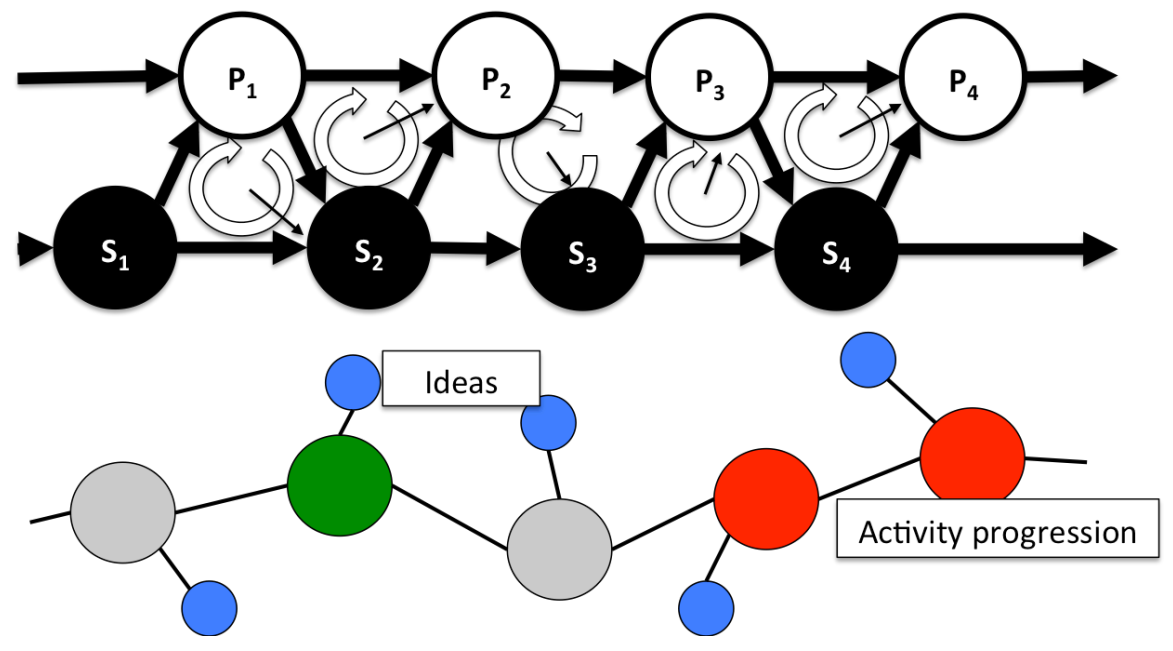

Figure 8b: Integrated and iterative ideation produces ideas that directly feed into and help drive the co-evolution process development. 
Figure 8: Integration of the ideation, problem solving, and design development co-evolution processes. Here, slim arrows represent ideas while ideation loops are denoted by in white. Each progression type is also illustrated with an example network segment.

The results also reveal a number of important insights that substantially extend our theoretical understanding of ideation processes. First, it is widely held that experienced practitioners produce fewer ideas because they have a more efficient ideation process (Ahmed, Wallace, and Blessing, 2003; Atman et al., 1999). However, the underlying mechanism for this 'efficiency' has proven difficult to describe (Atman et al., 1999). The approach presented here offers a means for decomposing this by bringing together the design development, problem solving, and ideation processes. Although the results are not generalisable, substantial differences were illustrated between the activity patterns and network growth of the teams in this respect. Of particular note is Team 2's focus on developing the design through rapidly linked iterations, each generating ideas that drive the next iteration. In contrast, Team 1 developed a large number of ideas but did not link them into the design development.

Previous works have offered partial explanations for improved design performance via parallel thinking (Seitamaa-Hakkarainen and Hakkarainen, 2001) or structured cognitive processes (Kavakli and Gero, 2002). Here, process interaction gives a new perspective on these phenomena. In particular the rapid, focused iteration between problem and solution spaces appears to be a key feature of the team's network growth. Team 2 illustrates this with each idea a product of iteration and a driver for the design development in a three way progression - deeply integrating idea generation and problem/solution co-evolution. Thus, it is possible to expand the work of Seitamaa-Hakkarainen and Hakkarainen (2001) into the technical domain and tie this parallel process concept directly to design development and ideation performance. Although Team 1 did not display the same degree of rapid iteration, 
they also progressed each of the observed elements in parallel, suggesting a more complex system than initially described by Seitamaa-Hakkarainen and Hakkarainen (2001). In particular the two teams illustrate an interesting phenomenon of process interaction. The rapid, focused, small scale iteration of Team 2 produced a highly linked sequence of activities closely coupling the three observed processes. Contrast this with Team 1 who used less iteration and were less focused on the design itself. Thus much of their ideation was carried out without reference to the design, and ideas were seldom used to drive the design progression. In this way they partially decouple the ideation and design development processes.

Finally, this work complements the Linkography approach originally proposed by Goldschmidt (1990). In Linkography design 'moves' are interrelated by 'links' describing any type of connection between individual moves. These connections are defined by the coder and can be based on theory or other rules (e.g. temporal connections). The ratio of links to moves and the number of links associated with individual moves can then be respectively used to give a measure of productivity or identify critical points in the design work (critical moves) (Kan and Gero, 2008). The approach described here gives a significantly expanded means for describing and tracing the multifaceted nature of designer activity via the network approach, and the possibility of using dynamic network analysis. This dynamic analysis can provide information on how networks (and thus process interactions in this case) develop in real time, paving the way for measurement and eventually prediction of network evolution. In particular, the proposed network approach allows for a wide range of link types to be described between various elements in the design process, moves, activities, ideas, objects, or artefacts, across multiple levels of granularity. This provides a more quantitatively accessible and dynamic means for exploring process interactions than current notation 
based approaches, such as that proposed by Sonalkar et al. (2013). For example, it is possible to link specific design tasks, methods and equipment (resources) used, and larger scale context or process stages. This ability to integrate a wide range of node and link types while retaining traceable differentiation between individual process progressions is a key differentiator from Linkography. Further, the possibility for applying dynamic measures of network growth allow for quantitative analysis of the modelled processes and interactions development, beyond that available in Linkography. In particular, this higher order analysis offers potential for re-assessment of studies using more traditional representations of activity interaction over time, such as Lee et al.'s (2014) examination of problem finding, solution-generation and solution-evaluation. As such, the described approach is complementary to Linkography, while allowing the researcher to extend their interpretation of design work to include multiple interacting perspectives and processes.

\section{Implications}

This section discusses the implications of this work for both researchers and practitioners.

First, the method and results help to bring together the different processes currently associated with design innovation in practice. This points to potential explanations for previously identified, but not fully explained phenomena, such as, differentiation between experts and novices (Ahmed et al., 2003; Seitamaa-Hakkarainen and Hakkarainen, 2001). In particular a relationship has been found between, design development, co-evolution, and ideation. This has important implications for future research efforts aiming to explain ideation activity in practice. Specifically the need for a focus on process orientated holistic understanding. 
Second, the results reinforce the importance of problem-solution co-evolution as not just an explanation of design work, but as a means of understanding and linking processes previously considered separately (Dorst and Cross, 2001). In particular the role of rapid iteration and design focus in driving effective ideation has been identified.

Third, the network approach provides a new means for decomposing and potentially measuring ideation activity and performance. This complements existing approaches by developing a process-based approach (Nelson et al., 2009). This allows the researcher to more effectively analyse protocol data, and leverage this to better explain previously irreducible process interactions. The fact that this allows for a multi process dynamic analysis, means that the researcher is, for the first time, able to effectively explore phenomena that are the result of process interactions rather than single process features.

\section{Conclusions and Opportunities for Further Research}

This paper has presented a new means for holistic assessment of ideation processes. In doing this significant new insights have been made into a number of features of ideation. The network approach to the holistic assessment of multiple related processes has offered a new perspective on ideation activity and highlighted its interrelation with other design processes. In particular the co-evolution of problem and solution understanding has been shown to be fundamental in explaining ideation processes.

This method gives researchers a new means for investigating ideation and other complex phenomena in the design and innovation context and substantially extends current approaches by offering an effective process-based assessment of ideation for the first time.

The study itself not only serves to illustrate the approach but also to highlight implications for innovation and ideation monitoring and management. In particular the 
iterative evolution of ideas in order to drive design development is a key finding. This is in contrast to a large number of methods that espouse non-reflective idea generation.

Based on this there are three distinct areas that are highlighted as important for further study. First, the relationship between ideation, design development, and social processes is a key next step in expanding the understanding of how the communication of ideas and design information impacts iteration and innovation performance. Second, there is scope for automation in the application of the network approach, particularly given the large number of extant protocol datasets. This could allow for significant reanalysis of existing data. Finally, there is a need for larger real world studies of ideation in order to map out possible inter-process patterns in addition to those identified here, and the individual process patterns typical of extant literature. This would allow innovation performance characteristics to be holistically linked to process interactions in order to support more targeted management of innovation methods.

\section{References}

Ahmed, S., and Storga, M. 2009. "Merged ontology for engineering design: Contrasting empirical and theoretical approaches to develop engineering ontologies". Al EDAM (Artificial Intelligence for Engineering Design, Analysis and Manufacturing), 23(4), 391.

Ahmed, S., Wallace, K. M., and Blessing, L. T. M. 2003. "Understanding the differences between how novice and experienced designers approach design tasks". Research in Engineering Design, 14(1), 1-11.

Atman, C. J., Chimka, J. R., Bursic, K. M., and Nachtmann, H. L. 1999. "A comparison of freshman and senior engineering design processes". Design Studies, 20(2), 131-152.

Baer, J. 2003. Evaluative Thinking, Creativity, and Task Specificity. In M. A. Runco (Ed.), Critical Creative Processes (pp. 129-151). Hampton Press, USA.

Bakeman, R., Deckner, D. F., \& Quera, V. (2003). Analysis of behaviour streams. In D. M. Teti (Ed.), Handbook of Research Methods in Developmental Psychology (pp. 394-421). Oxford, U.K.: Blackwell.

Barzelay, M. 1993. "The Single Case Study as Intellectually Ambitious Inquiry". The Journal of Public Administration Research and Theory, 3(3), 305-318. 
Briggs, R O, and B A Reinig. 2007. "Bounded Ideation Theory: A New Model of the Relationship Between Idea Quantity and Idea-Quality during Ideation." In 40th Hawaii International Conference on System Sciences, 1-10. Hawaii, January 3-6.

Cash, P, B Hicks, and S Culley. 2015. "Activity Theory as a Means for Multi-Scale Analysis of the Engineering Design Process: A Protocol Study of Design in Practice." Design Studies 38 (May): 132. doi:10.1016/j.destud.2015.02.001.

Cash, P., Hicks, B. J., and Culley, S. J. 2013. "A comparison of designer activity using core design situations in the laboratory and practice". Design Studies, 34(5), 575-611.

Cash, P., Stankovic, T., and Storga, M. 2014. "Using visual information analysis to explore complex patterns in the activity of designers". Design Studies, 35(1), 1-28.

Christiaans, H., and Venselaar, K. 2005. "Creativity in design engineering and the role of knowledge: Modelling the expert". International Journal of Technology and Design Education, 15(3), 217236.

Chulvi, V, E Mulet, A Chakrabarti, B López-Mesa, and C González-Cruz. 2012. "Comparison of the Degree of Creativity in the Design Outcomes Using Different Design Methods." Journal of Engineering Design 23 (4) (April): 241-269.

Clarkson, J. 2003. Inclusive Design: Design for the Whole Population. Springer Science \& Business Media.

Coughlan, T., and Johnson, P. 2006. "Interaction in Creative Tasks : Ideation, Representation and Evaluation in Composition". In SIGCHI Conference on Human Factors in Computing Systems (pp. 531-540). Canada, April 22-27.

Cross, N., Christiaans, H., and Dorst, K. 1996. Analysing design activity (p. 463). Chichester: John Wiley and Sons, UK.

Cross, N., and Cross, A. C. 1995. "Observations of teamwork and social processes in design". Design Studies, 16(2), 143-170.

D'Astous, P., Robillard, P. N., Detienne, F., and Visser, W. 2001. "Quantitative measurements of the influence of participant roles during peer review meetings". Empirical Software Engineering, 6(2), 143-159.

Design-Council. 2006. “Double Diamond Design Process Model [Online].” Accessed January 2015. http://www.designcouncil.org.uk/designprocess.

Dorst, K., and Cross, N. 2001. "Creativity in the design process: Co-evolution of problem-solution". Design Studies, 22(5), 425-437.

Finke, R. A., Ward, T. B., and Smith, S. M. 1992. Creative cognition: Theory, research, and applications (p. 239). Cambridge, Massachuetts: Cambridge, MA: MIT Press.

Flyvbjerg, B. 2006. "Five Misunderstandings About Case-Study Research". Qualitative Inquiry, 12(2), 219-245.

Gabora, L. 2002. "Cognitive mechanisms underlying the creative process". In Proceedings of the 4th conference on Creativity and cognition (pp. 126-133). New York, US.

Gero, J. S., and Kannengiesser, U. 2004. "The situated function-behaviour-structure framework". Design Studies, 25(4), 373-391.

Goldschmidt, G. 1990. "Linkography: Assessing Design Productivity." Cyberbetics and System '90.

Goodman-Deane, J, P Langdon, and J Clarkson. 2010. "Key Influences on the User-Centred Design Process." Journal of Engineering Design 21 (2): 345-373. 
Hagedorn, J, J Hailpern, and K G Karahalios. 2008. "VCode and VData: Illustrating a New Framework for Supporting the Video Annotation Workflow." In Conference on Advanced Visual Interfaces, 317-321. Italy, May 28-30.

Hanson, W. E., Creswell, J. W., Plano-Clark, V. L., Petska, K. S., and Creswell, J. D. 2005. “Mixed methods research designs in counselling psychology". Journal of Counseling Psychology, 52(2), 224-235.

Hatchuel, A., and Weil, B. 2003. A new approach of innovative design: An introduction to C-K theory. Stockholm, Sweden.

Howard, T. J., Culley, S. J., and Dekoninck, E. 2010. "Reuse of ideas and concepts for creative stimuli in engineering design". Journal of Engineering Design, 22(8), 565-581.

Kan, J. W. T., and Gero, J. S. 2008. "Acquiring information from linkography in protocol studies of designing". Design Studies, 29(4), 315-337.

Kavakli, M., and Gero, J. S. 2002. "The structure of concurrent cognitive actions: A case study on novice and expert designers". Design Studies, 23(1), 25-40.

Kirk, R. E. 2009. Experimental design (p. 800). London, UK: Sage Publications.

Kirton, M. 1976. "Adaptors and innovators: A description and measure". Journal of Applied Psychology, 61(5), 622-629.

Kitchenham, B. A. 1996. "Evaluating software engineering methods and tool part 1: The evaluation context and evaluation methods". ACM SIGSOFT Software Engineering Notes, 21(1), 11-14.

Kleinsmann, M., and Valkenburg, R. 2008. "Barriers and enablers for creating shared understanding in co-design projects". Design Studies, 29(4), 369-386.

Kudrowitz, B M, and D Wallace. 2013. "Assessing the Quality of Ideas from Prolific, Early-Stage Product Ideation." Journal of Engineering Design 24(2) (February): 120-139.

Lee, K-S, and K Lee. 2004. "Evolutionary Design and Re-Design Using Design Parameters and Goals." Journal of Engineering Design 15(2) (April): 155-176.

Lee, J H, N Gu, and M J Ostwald. 2014. "Creativity and Parametric Design? Comparing Designer's Cognitive Approaches with Assessed Levels of Creativity." International Journal of Design Creativity and Innovation 3 (2): 78-94.

Lopez-Mesa, B, E Mulet, R Vidal, and G Thompson. 2009. "Effects of Additional Stimuli on IdeaFinding in Design Teams." Journal of Engineering Design 22(1): 31-54.

Lopez-Mesa, B, E Mulet, R Vidal, and G Thompson. 2011. "Effects of Additional Stimuli on IdeaFinding in Design Teams." Journal of Engineering Design 22(1) (January): 31-54.

LiveScribe. 2011. LiveScribe: never miss a word. Accessed Januray 2015. http://www.livescribe.com/en-us/.

McAdam, R. 2004. "Knowledge creation and idea generation: a critical quality perspective". Technovation, 24(9), 697-705.

McDonnell, J., and Lloyd, P. 2009. About: Designing - Analysing design meetings. (J. McDonnell and P. Lloyd, Eds.) (p. 434). Boca Raton, FL. U.S.: CRC Press.

Nelson, B. A., Wilson, J. O., Rosen, D., and Yen, J. 2009. "Refined metrics for measuring ideation effectiveness". Design Studies, 30 (6), 737-743.

Newman, M. E. J. 2004. "Detecting Community Structure in Networks". The European Physical Journal B - Condensed Matter and Complex Systems, 38 (2), 321-330. 
Pahl, G, and W Beitz. 1996. Engineering Design: A Systematic Approach. London: Springer.

Purcell, A. T., and Gero, J. S. 1998. "Drawings and the design process: A review of protocol studies in design and other disciplines and related research in cognitive psychology". Design Studies, 19(4), 389-430.

Reinig, B. A., and Briggs, R. O. 2008. "On the relationship between idea-quantity and idea-quality during ideation". Group Decision and Negotiation, 17(5), 403-420.

Reinig, B. A., Briggs, R. O., and Nunamaker, J. F. 2007. "On the measurement of ideation quality". Journal of Management Information Systems, 23(4), 143-161.

Robson, C. 2002. Real World Research. Vol. 2nd. Chichester: Wiley.

Sawyer, R. K. 2003. Group creativity: Music, theater, collaboration (p. 214). New York, US: Routledge.

Schlecht, L, and M Yang. 2014. "Impact of Prototyping Resource Environments and Timing of Awareness of Constraints on Idea Generation in Product Design." Technovation 34 (4) (April): 223-231. doi:10.1016/j.technovation.2013.11.001.

Seitamaa-Hakkarainen, P., and Hakkarainen, K. 2001. "Composition and construction in experts' and novices' weaving design". Design Studies, 22(1), 47-66.

Shah, J. J., Smith, S. M., and Vargas-Hernandez, N. 2003. "Metrics for measuring ideation effectiveness". Design Studies, 24(2), 111-134.

Simonton, D. K. 2003. "Scientific creativity as constrained stochastic behavior: The integration of product, person, and process perspectives". Psychological Bulletin, 129(4), 475-494.

Smith, R. P., and Tjandra, P. 1998. "Experimental observation of iteration in engineering design". Research in Engineering Design, 10(2), 107-117.

Snider, C. 2014. "Characterising the Creative Behaviour of Designers within the Late-Stage Engineering Design Process." University of Bath.

Sonalkar, N, A Mabogunje, and L Leifer. 2013. "Developing a Visual Representation to Characterize Moment-to-Moment Concept Generation in Design Teams." International Journal of Design Creativity and Innovation 1 (2): 93-108.

Spitas, C. 2011. "Analysis of Systematic Engineering Design Paradigms in Industrial Practice: A Survey." Journal of Engineering Design 22 (6) (June): 427-445.

Stankovic, T, M Storga, I Stojic, and T Savsek. 2012. "Traceability Visualisation Toolkit." Design 2012 conference. Croatia, May 21-24.

Stempfle, J., and Badke-Schaub, P. 2002. "Thinking in design teams-an analysis of team communication". Design Studies, 23(5), 473-496.

Stones, C., and Cassidy, T. 2010. "Seeing and discovering: how do student designers reinterpret sketches and digital marks during graphic design ideation?" Design Studies, 31, 439-460.

Storga, M., Andreasen, M. M., and Marjanovic, D. 2010. "The design ontology: Foundation for the design knowledge exchange and management". Journal of Engineering Design, 21(4), 427-454.

Tang, H. K. 1998. "An integrative model of innovation in organizations". Technovation, 18(5), 297309.

Torrance, E. P. 1968. Torrance tests of creative thinking. Personnel Press, Inc.

Torrance, E. P. 1998. Torrance tests of creative thinking: Norms-technical manual: Figural (streamlined) Forms $A$ and B. Scholastic Testing Service. 
Torrance, E. P. 2007. Torrance tests of creative thinking: Norms-technical manual: Figural (streamlined) Forms $A$ and B. Scholastic Testing Service.

Torrance, E. P. 2008. Torrance Test of Creative Thinking: Norms-Technical Manual Figural (Streamlined) Forms A and B. Bensenville, IL: Scolastic Testing Service Inc.

Ulrich, K T, and S D Eppinger. 2003. Product Design and Development. Vol. 5th. New York, USA: MvGraw-Hill.

Vargas-Hernandez, N., Shah, J. J., and Smith, S. M. 2010. "Understanding design ideation mechanisms through multilevel aligned empirical studies". Design Studies, 31(4), 382-410.

Wallas, G. 1926. The art of thought. (p. 204). New York, US: New York: Harcourt.

Wasiak, J., Hicks, B. J., Newnes, L., Dong, A., and Burrow, L. 2010. “Understanding engineering email: the development of a taxonomy for identifying and classifying engineering work". Research in Engineering Design, 21(1), 43-64. 